\title{
DISEÑO DE UN BANCO PARA LA DETERMINACIÓN PRÁCTICA DEL COEFICIENTE DE VÁLVULAS, NORMA VDI/VDE 2173
}

\section{Training bench design for practical valve coefficient determination, standard VDI/VDE 2173}

\author{
César Zambrano Miranda ${ }^{1}$, José Alonso González ${ }^{1}$, Luis Campo Escudero ${ }^{1}$, Ricardo Araújo Segura ${ }^{1}$, \\ Javier Roldán Mckinley ${ }^{2}$ \\ ${ }^{1}$ Ing. Universidad del Atlántico (Barranquilla, Colombia). cesarz503@hotmail.com, \\ jose frag@hotmail.com, luisfernando.campo@gmail.com, ricardo1924@hotmail.com \\ ${ }^{2}$ Ph.D. Universidad del Atlántico (Barranquilla, Colombia). javierroldan@mail.uniatlantico.edu.co
}

(Recibido septiembre 21 de 2017 y aceptado noviembre 08 de 2017)

\begin{abstract}
Resumen
Se presenta el proceso de diseño de un banco de entrenamiento para la determinación del coeficiente de flujo de válvulas de control, usando las indicaciones de la norma VDI/VDE 2173, que establece parámetros para la elaboración de este tipo de ensayos. Se adopta un criterio de ergonomía para el dimensionamiento preliminar del banco con base en datos de la antropometría colombiana. Los elementos estructurales del equipo son posteriormente diseñados mediante la comparación de cálculos de teoría de falla con análisis de elemento finito en SolidWorks.
\end{abstract}

Palabras clave: Ergonomía; diseño estructural; banco de entrenamiento; coeficiente de flujo; SolidWorks.

\begin{abstract}
It is presented the design of a training bench for determining the flow coefficient of control valves, based on standard VDI/VDE 2173 that sets indications for this test. Preliminary sizing of the bench follows ergonomic considerations, taking into account the Colombian population anthropometrics. Structural elements of the device are designed by contrasting both failure analysis equations with finite element analysis results using SolidWorks.
\end{abstract}

Key words: Ergonomics; structure design; test bench; flow coefficient; SolidWorks.

\section{INTRODUCCIÓN}

El continuo cambio en las tecnologías y el planteamiento de conceptos requeridos para explicar la aparición de fenómenos, requieren que el Ingeniero Mecánico se prepare un cambiante escenario [1], más allá de la formulación teórica hasta la aplicación de los conocimientos adquiridos, con herramientas tecnológicas en la resolución de los problemas, dentro de las cuales el uso de equipos de laboratorio cada día cobra mayor importancia en la formación académica del futuro profesional. Aunque las primeras propuestas en el uso de herramientas de laboratorio para la enseñanza práctica fueron expuestas por Jhon Locke [2] hace más de tres siglos, hoy resultan casi que indispensables para afianzar la enseñanza de un aprendiz. El desarrollo de prácticas de laboratorio influencia positivamente el profesionalismo y la competitividad del estudiante, ayuda a optimizar el tiempo en el desarrollo de actividades, afianza su capacidad cognitiva y fortalece su relación con otros individuos logrando mayor eficacia en trabajo grupales [3].

Actualmente, la instrumentación industrial brinda un gran soporte para llevar a cabo tareas de automatización y control, por lo cual se requiere que el Ingeniero Mecánico egrese con conocimiento de la simbología de procesos (P\&ID por sus siglas en inglés de Piping and Instrumentation Diagram) y con el conocimiento 
teórico-práctico de sus elementos: bombas, tuberías, tanques de almacenamiento, válvulas, sensores y circuitos hidráulicos básicos, entre otros [4]. Dentro de esta disciplina, el dimensionamiento y estudio de las válvulas de flujo ocupa un lugar fundamental por tratarse de los instrumentos primarios o en contacto directo con el proceso, siendo el concepto del coeficiente de flujo mediante el cual se cuantifica el desempeño y requerimientos de este instrumento.

El coeficiente de flujo, $\mathrm{Kv}$, se define como el caudal de agua, entre $5^{\circ} \mathrm{C}$ y $40^{\circ} \mathrm{C}$, en metros cúbicos por hora $\left[\mathrm{m}^{3} / \mathrm{h}\right.$ ] que pasa a través de una válvula a una apertura dada y con una pérdida de carga de 1 bar, y se simboliza con las letras $K_{\mathrm{V}}$ [5]. En la industria es muy frecuente encontrar montajes para la determinación del coeficiente de flujo para grandes válvulas en la misma línea de operación donde se encuentran instaladas, lo cual incrementa el costo del análisis del elemento. De otra parte, el uso de estándares para este tipo de estudios resulta beneficioso al momento de obtener buenos resultados en las mediciones, normas como la IEC 60534-2-3 [6] y la VDI/VDE 2173 [7], agrega valor significativo en la determinación del coeficiente de flujo, brindando información tanto en la disposición de elementos para llevar a cabo la prueba como en el proceso analítico de los resultados.

En [8] y [9] se documenta el análisis del coeficiente de flujo en válvulas, donde se obtienen óptimos resultados, aunque resulta evidente que no se han aplicado las normas especializadas en la determinación del coeficiente, sea para el montaje de elementos o para calcular el coeficiente, además se aprecia la carencia del diseño bajo criterios de ergonomía que favorezca la fácil operación del equipo, evitando posiciones de trabajo forzosas e inadecuadas. En el mercado se encuentran compañías especializadas en el desarrollo de equipos de laboratorio con un alto grado de calidad en sus productos. GUNT Hamburg [10] es una empresa reconocida en elaborar equipos para la educación en ingeniería, que dispone de un equipo para la determinación del coeficiente de flujo, con elementos de calidad y software especializado en el análisis, pero también se logra evidenciar la falta de un diseño ergonómico.
En este artículo se presenta el diseño de un banco de pruebas o de entrenamiento para la determinación práctica del coeficiente de válvulas de hasta media pulgada. Se consideran aspectos de ergonomía y seguridad estructural del equipo, principalmente, para crear un diseño que sea de uso cómodo y confiable. Dado el alto costo de estos equipos por parte de las empresas internacionales, se hace pertinente la aplicación de estos conceptos para generar un producto nacional de bajo costo que puede utilizarse para reforzar la enseñanza en las universidades y para la venta de servicios especializados.

\section{ASPECTOS DE DISEÑO}

El diseño del banco de pruebas está determinado por tres factores fundamentales: diseño red de tuberías, ergonomía en el diseño y diseño estructural. El primero de estos se considera el más importante y está sujeto a la descripción brindada por los estándares internacionalmente utilizados para la determinación del coeficiente de flujo.

\subsection{Diseño de red de tuberías}

Las normas IEC 60534-2-3 [6] y VDI/VDE 2173 [7] describen las pautas para la realización del procedimiento de obtención de un valor de coeficiente de flujo de forma experimental, utilizando un conjunto de elementos que permiten controlar las condiciones mencionadas, y proponen un esquema básico para la realización del procedimiento para una válvula de control, como en la figura 1.

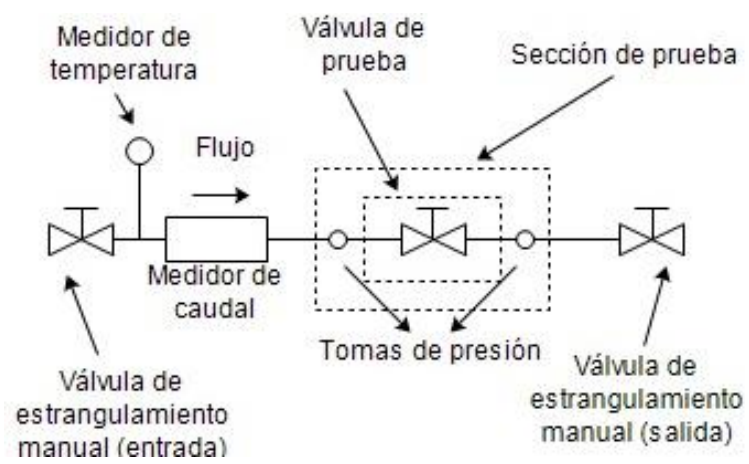

Figura 1. Esquema básico según norma.

La ubicación de las tomas de presión guarda una relación proporcional con el diámetro nominal de la válvula de pruebas, las distancias entre 
elementos dependerán estrictamente del tamaño de la válvula. En la figura 2 se muestra un esquema para la ubicación de estos elementos, donde $L_{1}: 2$ veces el diámetro nominal de la tubería, $\mathrm{L}_{2}: 6$ veces el diámetro nominal de la tubería, $\mathrm{L}_{3}$ : es por lo menos 18 veces el diámetro nominal de la tubería y $\mathrm{L}_{4}$ : al menos 1 vez el diámetro nominal de tubería. La longitud del tubo aguas arriba, debe ser por lo menos 20 veces el diámetro y aguas abajo debe ser por lo menos 7 veces el diámetro nominal del tubo. Para el suministro del fluido, se utiliza una bomba centrífuga seleccionada de acuerdo a los requerimientos del diseño de la red de tuberías.

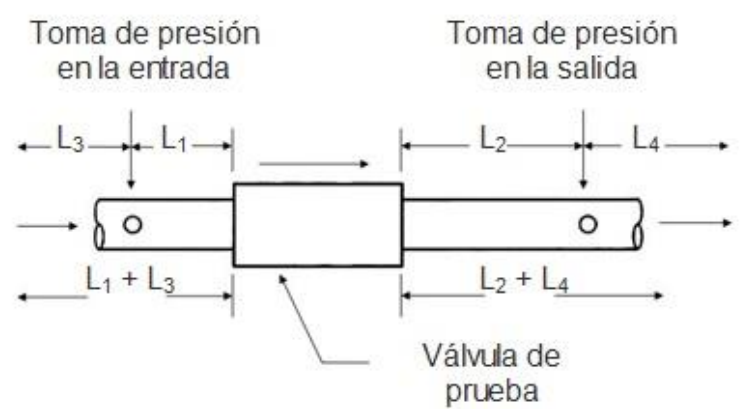

Figura 2. Configuración de toma de presiones.

A partir de la información brindada en [6] y [7] se ha diseñado el P\&ID para el banco de pruebas mostrado en la figura 3 , formado por un conjunto de tuberías, accesorios y elementos con una configuración en circuito cerrado, que a su vez incluye un tanque de almacenamiento de agua y una bomba que hace circular el fluido. A partir de criterios ergonómicos y el valor de $Z$ se limita a la altura de maniobrabilidad del banco, lo que representa un máximo de $102 \mathrm{~cm}$. De acuerdo a lo anterior, se ha establecido un valor aproximado para $Z$ de $62 \mathrm{~cm}$, dejando espacio suficiente para la correcta instalación de la bomba.

La válvula seleccionada para llevar a cabo la prueba es la válvula Danfoss EV260B-15B [11] que cuenta con un coeficiente de flujo $K v=2.1 \mathrm{~m}^{3} / \mathrm{h}$, conexión de $1 / 2$ " NPT y accionamiento mediante un actuador solenoide. Se ha optado por utilizar tubería en PVC, ya que este tipo de material reúne ventajas que van desde la resistencia a la corrosión y a ataques químicos, hasta un acabado liso en su interior que reduce considerablemente la pérdida por fricción en el sistema. Se han seleccionado dos diámetros diferentes para la tubería. La entrada de la electrobomba tiene tubería con diámetro nominal de 1 in $(25 \mathrm{~mm})$ con resistencia de hasta 500 PSI. Para la salida de la bomba y el resto del circuito, se ha seleccionado una tubería de $1 / 2$ in $(15 \mathrm{~mm})$ de diámetro nominal con resistencia de hasta 500 PSI.

Para la selección de la bomba de suministro de agua es necesario conocer las pérdidas del sistema mostrado en la figura 3 , incluyendo pérdidas principales y por accesorios. Se establecen los puntos $a$ y $b$ en la figura 3 como base para formular la ecuación (1) que describe la ecuación general de la energía [12].

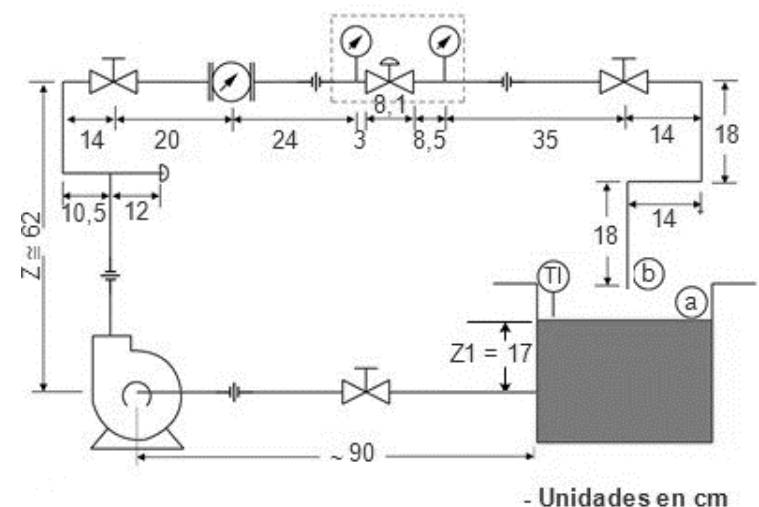

Figura 3. Diagrama P\&ID del banco de pruebas.

$\frac{\mathrm{P}_{\mathrm{A}}}{\delta}+\mathrm{Z}_{1}+\frac{\mathrm{V}_{\mathrm{A}}^{2}}{2 \mathrm{~g}}+\mathrm{h}_{\mathrm{A}}-\mathrm{h}_{\mathrm{R}}-\mathrm{h}_{\mathrm{L}}=\frac{\mathrm{P}_{\mathrm{B}}}{\delta}+\mathrm{Z}_{2}+\frac{\mathrm{V}_{\mathrm{B}}^{2}}{2 \mathrm{~g}}(1)$

De acuerdo al sistema en la figura 3 , algunos términos de la ecuación (1) se cancelan: $P_{A}=0$, superficie del depósito de agua expuesto a la presión atmosférica; $P_{B}=0$, corriente en la salida de la tubería expuesta a la presión atmosférica; $V_{A}=0$, el área de la superficie del depósito es relativamente grande en comparación al área de tubería de succión; $h_{R}=0$, al no existir un dispositivo mecánico que remueva energía al fluido. Para encontrar la pérdida total de energía del sistema $h_{A}$ de la ecuación (1); el diámetro de la línea de succión es diferente al diámetro de la línea de descarga, por lo cual las pérdidas de energía en el sistema $h_{L}$ se calculan separadamente para cada tubería. Se simplifican cálculos al expresar la ecuación (1) en función del caudal, como en la ecuación (2).

Las longitudes equivalentes totales $L_{\text {eq }}$ en la línea de succión y la línea de descarga están dados por la 
suma total de cada $L_{\text {eq }}$ por accesorio [13]. Se determinan las longitudes equivalentes $L_{E Q 1}$ (línea de succión) y $\mathrm{L}_{\mathrm{EQ} 2}$ (línea de descarga) para los tramos de tubería según la cantidad determinada de accesorios incluidos en el P\&ID de la figura 3. Dentro de los accesorios se destaca la válvula de pruebas como un elemento que, para un $\mathrm{K}_{\mathrm{v}}$ de 2.1 $\mathrm{m}^{3} / \mathrm{h}$ (fabricante), cuenta con una perdida equivalente de $10.6 \mathrm{~m}$ [14].

La bomba se selecciona mediante la intersección dos curvas: una que incluye todos los elementos encontrados en la red de tuberías, y otra donde se excluya solo la válvula de pruebas, encontrando el punto de operación del sistema y un valor estimado de la presión diferencial que hay en la válvula de pruebas. Para encontrar ambas curvas se aplica la ecuación (2), donde solo tendrá la variación del valor de $\mathrm{L}_{\mathrm{EQ} 2}$. Para la curva del sistema principal (que incluye todos los elementos) se tiene que $L_{E Q 2}($ total) $=18.96 \mathrm{~m}[13,14]$, y para la curva sin la válvula de pruebas una longitud equivalente de $L_{E Q_{2}}(\sin$ válvula $)=8.36 \mathrm{~m}$ [13], donde esta última está determinada por la diferencia entre $\mathrm{L}_{\mathrm{EQ}_{2}}$ (total) y $\mathrm{L}_{\mathrm{EQ} 2}$ (sin válvula). En la figura 4 se muestran las curvas del sistema obtenidas a partir de la ecuación (2).

$$
\begin{aligned}
h_{A}= & Z_{2}-Z_{1}+\frac{V_{B}^{2}}{2 g}+\left(f_{1} \frac{L_{1}}{d_{1}^{5}} * \frac{8 Q^{2}}{\pi^{2} g}+f_{1} \frac{L_{e q 1}}{d_{1}^{5}} * \frac{8 Q^{2}}{\pi^{2} g}\right) \\
& +\left(f_{2} \frac{L_{2}}{d_{2}^{5}} * \frac{8 Q^{2}}{\pi^{2} g}+f_{2} \frac{L_{e q 2}}{d_{2}^{5}} * \frac{8 Q^{2}}{\pi^{2} g}\right)
\end{aligned}
$$

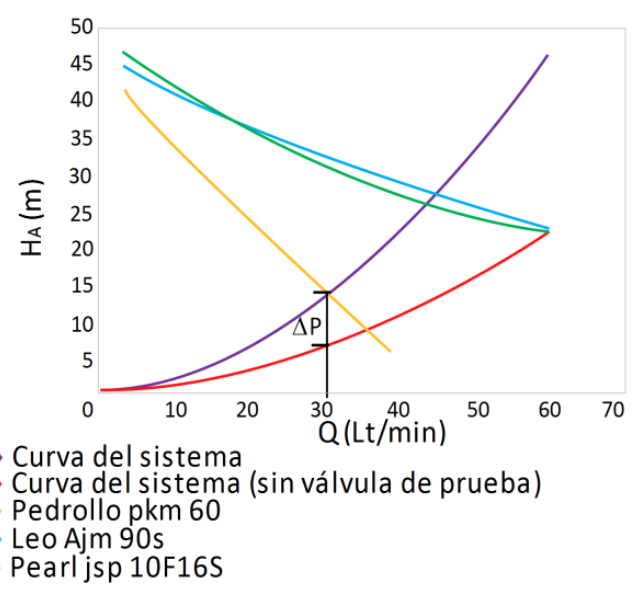

Figura 4. Punto de operación, diferentes bombas.

En el estudio del coeficiente de flujo es conveniente suministrar un caudal superior al valor del coeficiente de flujo de la válvula, y cuya presión diferencial entre la altura manométrica de la curva del sistema y la curva del sistema sin la válvula de prueba, sea superior a 1 bar (10.2 m.c.a.). La válvula de control Danfoss EV260B-15B seleccionada para la prueba tiene un $K_{v}$ de 2.1 $\mathrm{m}^{3} / \mathrm{h}$ (35 L/min). Es importante considerar el límite de velocidad del fluido permitido en tuberías para alargar la vida útil de las tuberías. En el caso de tuberías construidas en PVC el límite de velocidad es de $5.0 \mathrm{~m} / \mathrm{s}$ [15]. Con este valor se calcula el caudal para tuberías de PVC de $1 / 2$ in, obteniendo $3.18 \mathrm{~m}^{3} / \mathrm{h}(53.1 \mathrm{~L} / \mathrm{min})$.

Se selecciona la bomba Leo AJm90s [16] (ver figura 4) que proporciona un caudal aproximado de 44 L/min, con una presión diferencial aproximada de 1.56 bar $(15.9 \mathrm{~m})$ cumpliendo con las características para el funcionamiento del sistema. La bomba tiene un peso de $11 \mathrm{~kg}$, cuerpo elaborado en acero inoxidable e impulsor plástico de alta resistencia, que ayuda a evitar la corrosión y el desprendimiento de partículas que puedan afectar y deteriorar los demás elementos del sistema de tuberías. El dimensionamiento del tanque se hace de acuerdo al valor de Z1 dado en la figura 3, diseñado de manera que se mantenga el nivel recomendado. Se estima utilizar para la línea un volumen de agua entre $35 \mathrm{~L}$ y $45 \mathrm{~L}$, por lo cual se diseña el depósito con capacidad máxima $50 \mathrm{~L}$, de dimensiones: altura $350 \mathrm{~mm}$, anchura $360 \mathrm{~mm}$ y profundidad $400 \mathrm{~mm}$.

\subsection{Diseño ergonómico}

Siendo un banco de entrenamiento un elemento que tendrá contacto directo con el usuario, la accesibilidad al equipo y sus elementos es un factor muy importante, por lo cual las dimensiones del banco no deben ser ajenas a las dimensiones del cuerpo humano. En este sentido, se deben considerar aspectos ergonómicos en la interacción de los usuarios y el banco, tratando de brindar bienestar y comodidad durante el uso del banco [17]. Además de tener en cuenta la postura de trabajo, se han analizado las medidas antropométricas promedio (percentil 50) para la población colombiana [18], y las diferentes variables para el diseño, que incluyen la importancia de determinar una altura para la operación (plano de trabajo), área de trabajo, y la altura del dispositivo de visualización con respecto al suelo, con el fin de determinar la ubicación de 
una pantalla. Las medidas antropométricas a comparar son: la altura acromial (de pie), longitud alcance lateral con asimiento, alcance anterior con asimiento, altura radial (altura del codo), altura de ojos y anchura biacromial que, según [18], tienen unas dimensiones de $132.5 \mathrm{~cm}, 73.5 \mathrm{~cm}, 68.5 \mathrm{~cm}$, $102.3 \mathrm{~cm}, 151.5 \mathrm{~cm}$ y $37.4 \mathrm{~cm}$, respectivamente.

2.2.1 Altura de plano de trabajo. El tipo de trabajo en el uso de un banco de ensayos se considera trabajo ligero, lo que sugiere que la altura del plano de trabajo esté entre 0 y $10 \mathrm{~cm}$ por debajo de la altura del codo. Se ha tomado como referencia la altura del codo, que para una posición cómoda corresponde a brazo y antebrazo separados aproximadamente 90 grados [19]. El diseño del banco se hará para el promedio, teniendo en cuenta la media aritmética y el percentil 50 , que representan una medida de tendencia central para por lo menos el $50 \%$ de los usuarios, como resultado se obtiene una altura de plano de trabajo de $102.3 \mathrm{~cm}$ [18]. El banco estará soportado por ruedas para facilitar movilidad dentro del laboratorio o aula, estas tienen una altura que oscila entre los 9 y $11 \mathrm{~cm}$, añadiendo altura al plano de trabajo para una altura aproximada de $102 \mathrm{~cm}$ como se muestra en la figura 5.

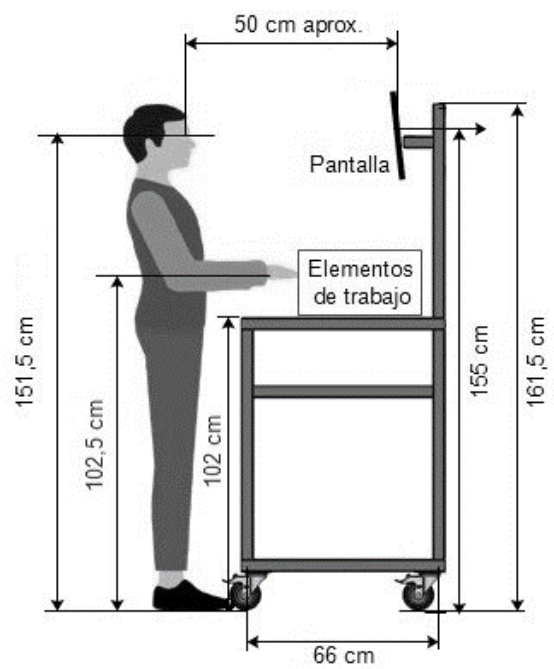

Figura 5. Altura y profundidad del monitor.

2.2.2 Área de trabajo. En el cálculo del área de trabajo se debe tener en cuenta la longitud de alcance lateral con asimiento (agarre) y el alcance anterior, siendo para el P50, de $73.5 \mathrm{~cm}$ y $68.5 \mathrm{~cm}$, respectivamente. Simultáneamente, se requiere respetar una serie de medidas para la instalación de elementos que conformarán el sistema, distancias que se encuentran soportadas con la norma VDI/VDE 2173 [7]; el banco debe tener una longitud mínima de $127 \mathrm{~cm}$, como se estableció en el análisis del P\&ID. De la diferencia entre la longitud de alcance lateral de asimiento y la anchura biacromial, resulta la longitud promedio del brazo con un valor de $54.8 \mathrm{~cm}$, el mismo alcance máximo de trabajo. Se estableció entonces un ancho total para el banco de $170 \mathrm{~cm}$, de manera que el área restante le sirva al operario para ubicar implementos de estudio: textos, computadora, guías, etc., sin que estos obstaculicen la manipulación del banco. La figura 6 muestra la distribución de longitudes para el área de trabajo.

2.2.3 Altura del monitor. Se ha incluido una pantalla para la adecuada visualización del proceso de operación del banco de pruebas. La altura y ubicación correcta de la pantalla se determina con la variable antropométrica altura de los ojos, la cual, mirando a $0^{\circ}$ y para el $\mathrm{P} 50$, se tiene un valor de $151.5 \mathrm{~cm}$. Se estableció una altura máxima para la estructura de $161.5 \mathrm{~cm}$, altura suficiente para la ubicación de una pantalla, donde la posición central de esta última, estará a una altura de 155 $\mathrm{cm}$ con respecto a la superficie del piso. La distancia entre la vista del operario y la pantalla se recomienda en $50 \mathrm{~cm}$ [20]; a partir de este valor e incluyendo el soporte de fijación de la pantalla, se ha estimado una longitud de profundidad para el banco de pruebas de $66 \mathrm{~cm}$. En la figura 5 se muestran la altura máxima del banco de pruebas y la posición ideal para la pantalla de visualización.

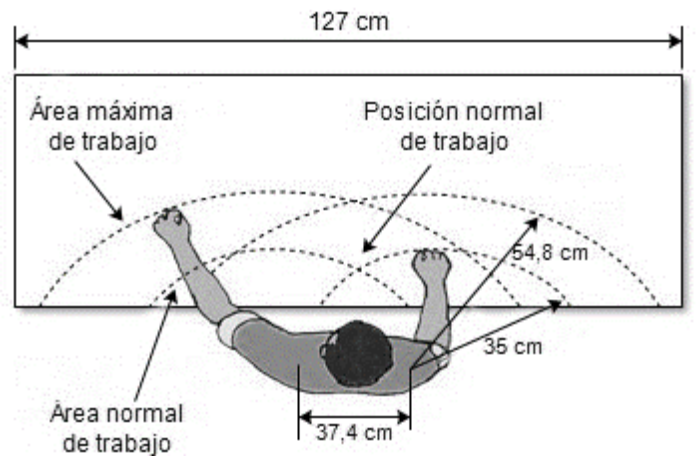

Figura 6. Área de trabajo.

\subsection{Diseño Estructural}

El diseño estructural del banco de pruebas se basa en el esquema mostrado en la figura 3 , en 
combinación con las dimensiones anteriormente establecidas. La estructura debe ajustarse de tal forma que facilite la maniobrabilidad y ser capaz de soportar el peso de los elementos y fuerzas externas. Es necesario realizar un análisis estructural con el fin de comprobar que la estructura no presente fallos en sus elementos. Para el análisis se toma como cargas el peso de los elementos descritos en el diseño de red y, adicionalmente se aplica una carga hipotética con el fin de garantizar un factor de seguridad confiable. Se ha escogido una estructura tubular cuadrada como se ilustra en la figura 7.

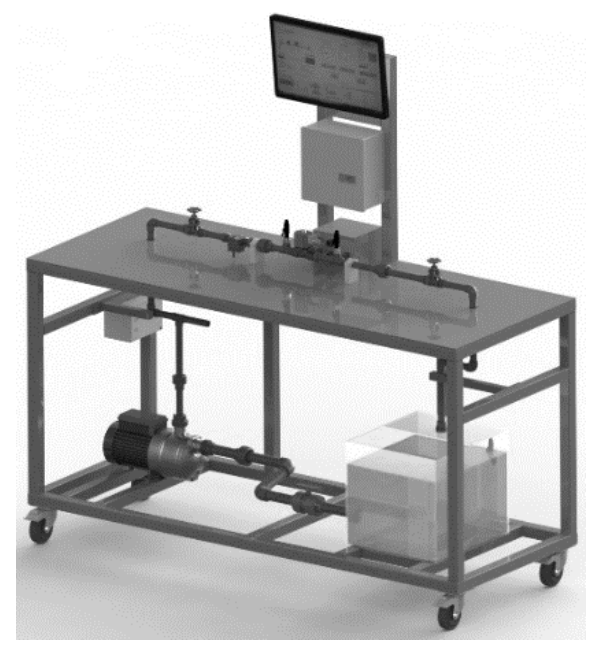

Figura 7. Estructura y elementos del banco, CAD.

El análisis estructural para el caso estático combina cálculos de teoría de falla y análisis de elementos finitos en SolidWorks. Para el cálculo se tienen las siguientes cargas: bomba (11 kg) $107.91 \mathrm{~N}$, depósito de agua (40 kg) $382.4 \mathrm{~N}$, tubería, accesorios y elementos electrónicos (7.67 kg aprox.) $75.24 \mathrm{~N}$, y una carga hipotética (230 kg) 2256.3 N. Estas cargas se distribuyen como se observa en la figura 8, donde la carga que actúa en la parte superior del banco será la suma de la carga hipotética más la carga por accesorios (2331.54 N). Para efectos de diseño, la carga aplicada en la cara superior, se considerará como distribuida. El valor de la carga distribuida se obtiene dividiendo la carga puntual total aplicada en la parte superior entre la longitud equivalente $(5.8 \mathrm{~m})$ de los elementos de la cara superior de la estructura, resultando en una carga distribuida $P_{h}=402 N / m$. En la figura 9 se muestran las dimensiones de la estructura del banco de pruebas.

2.3.1 Análisis estático de la estructura. Del diseño de la estructura se asume que el elemento viga IM en la figura 8 es crítico, por soportar las cargas procedentes de las 4 columnas posteriores, y las cargas aplicadas en los soportes de la bomba y del depósito de agua. Con esta conjetura, se omite el cálculo de los momentos flectores para los otros elementos estructurales. Se procede con el análisis estático para cada elemento y determinar las cargas en cada nodo. A partir de las dimensiones mostradas en la figura 9, se construye el diagrama de cuerpo libre para el elemento $A B$, figura 10 , el cual soporta una carga distribuida de $402 \mathrm{~N} / \mathrm{m}$. Aplicando análisis estático se obtienen los valores para las reacciones en los apoyos, donde $R_{A 1}=R_{B 1}=124.62 \mathrm{~N}$.

Por simetría de la estructura, los elementos $C D, E F$ Y GH, figura 8, están sometidos a la misma carga de $A B$ con un valor de $402 \mathrm{~N} / \mathrm{m}$, obteniendo para las reacciones: $\quad \mathrm{R}_{\mathrm{A} 1}=\mathrm{R}_{\mathrm{B} 1}=\mathrm{R}_{\mathrm{C} 1}=\mathrm{R}_{\mathrm{D} 1}=\mathrm{R}_{\mathrm{E} 1}=$ $R_{F 1}=R_{G 1}=R_{H 1}=124.62 \mathrm{~N}$. La figura 11 muestra la distribución de cargas para el elemento $\mathrm{BH}$. Las cargas están distribuidas simétricamente por lo tanto el valor de las reacciones será la carga total dividida entre 2, por lo cual $\mathrm{R}_{\mathrm{B} 2}=\mathrm{R}_{\mathrm{H} 2}=458.28 \mathrm{~N}$.

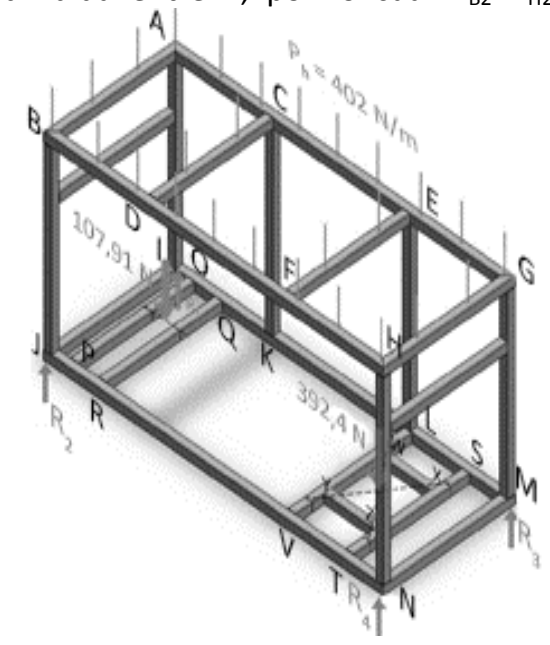

Figura 8. Distribución de carga y reacciones. 


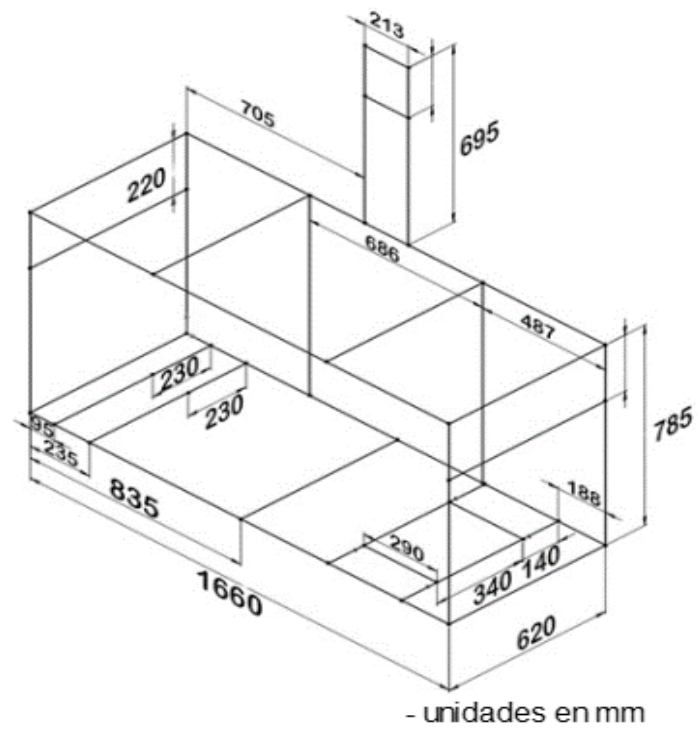

Figura 9. Dimensiones estructura banco de pruebas.

El elemento $A G$, figura 8 , se analiza como un pórtico plano debido a que las columnas $\mathrm{CK}$ y $\mathrm{EL}$ están apoyadas en el elemento IM y no en el suelo. La figura 12 muestra la distribución de cargas en el pórtico. Con la ayuda de un software para análisis estructural, se haya el valor de las reacciones en las columnas $\mathrm{Al}, \mathrm{CK}, \mathrm{EL}$ Y GM, resultando el diagrama de cortantes de la figura 13, mostrando $R_{A 2}=224.9$ $\mathrm{N}, \mathrm{R}_{\mathrm{C} 2}=118.57 \mathrm{~N}, \mathrm{R}_{\mathrm{E} 2}=81.8 \mathrm{~N}$ y $\mathrm{R}_{\mathrm{G} 2}=24.91 \mathrm{~N}$.

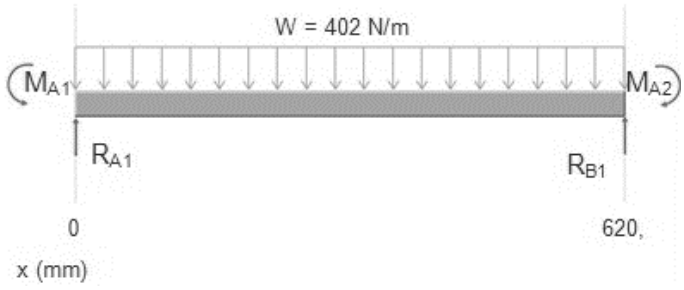

Figura 10. Diagrama de cuerpo libre elemento AB.

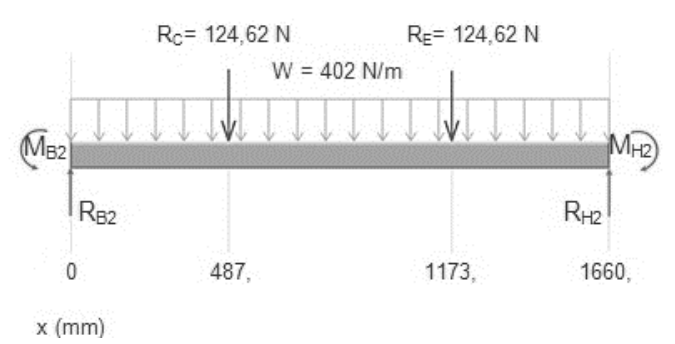

Figura 11. Diagrama de cuerpo libre elemento BH.
Se conocen los valores de todas las reacciones en los nodos de la parte superior, por lo cual es posible conocer cuál de las columnas está sometida a la mayor carga. Se tiene $R_{A}=R_{A 1}+R_{A 2}=349.61 \mathrm{~N}$, $R_{B}=R_{B 1}+R_{B 2}=582.9 \quad \mathrm{~N}, \quad R_{C}=R_{C 1}+R_{C 2}=243.19 \quad N$, $R_{E}=R_{E 1}+R_{E 2}=206.42 \mathrm{~N}, R_{G}=R_{G 1}+R_{G 2}=366.53 \mathrm{~N} \mathrm{y} R_{H}=$ $R_{\mathrm{H} 1}+R_{\mathrm{H} 2}=582.9 \mathrm{~N}$. Se observa que las cargas más altas corresponden a $R_{B}$ y $R_{H}$. Se escoge la carga $R B$ actuante sobre la columna $B J$ para hacer un análisis de columna, con el fin de establecer si la estructura presentará fallo por pandeo o puede llegar a colapsar.

La carga RB actúa verticalmente sobre la columna BJ, figura 14. La estructura presentará fallo por pandeo si la carga en la columna supera la carga crítica, la cual depende del tipo de columna: corta o larga. Para identificar el caso del tipo de columna, se compara la relación de esbeltez y la relación de esbeltez de transición. Se aplica la ecuación (3) para calcular la relación de esbeltez [21] $R_{\text {ee, }}$ para la columna $\mathrm{BJ}$, donde $\mathrm{K}$ es el factor de longitud efectiva, $L$ es la longitud del elemento, y $r_{\min }$ es el radio de giro mínimo de la sección transversal de la columna calculada mediante la ecuación (4) [21], donde I es el momento de inercia de la sección y $A$ es su área.

$$
\begin{aligned}
& R_{e e}=\frac{K L}{r_{\min }} \\
& r=\sqrt{\frac{I}{A}}
\end{aligned}
$$

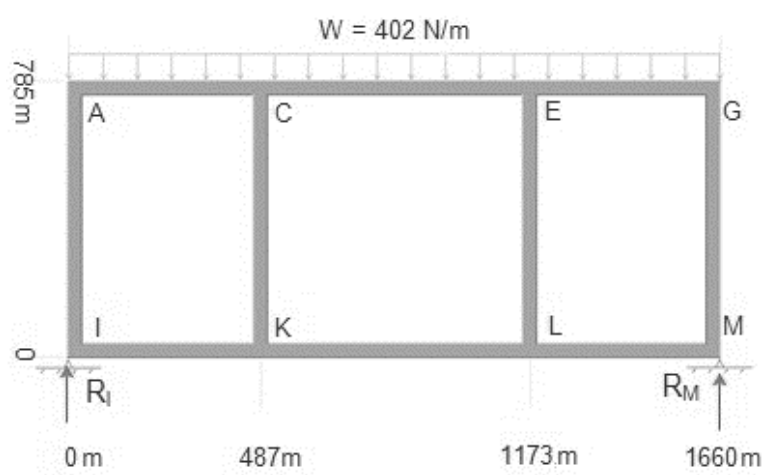

Figura 12. Diagrama de cuerpo libre elemento AG. 


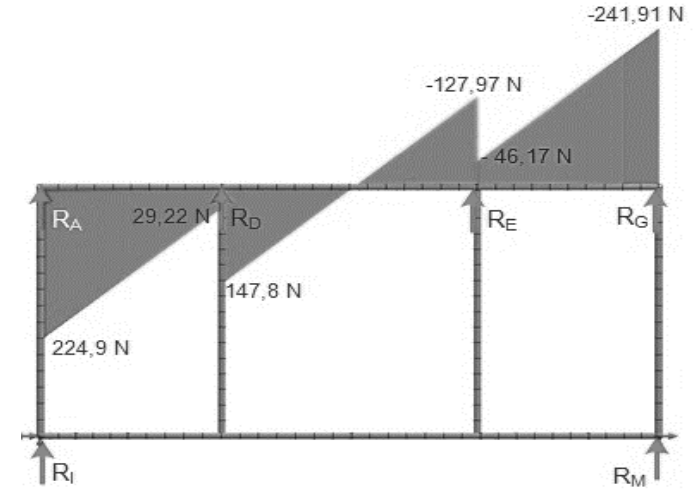

Figura 13. Diagrama de fuerzas cortantes elemento AG.

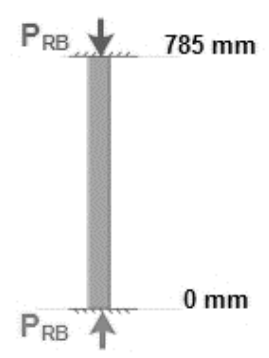

Figura 14. Diagrama de cuerpo libre elemento BJ.

El banco se evalúa con tubo cuadrado de $40 \mathrm{~mm}$, espesor de pared de $2.5 \mathrm{~mm}\left(\mathrm{I}=88281.2 \mathrm{~mm}^{4}\right.$, A $=375 \mathrm{~mm}^{2}$ ) material A36. Para la condición de carga $\mathrm{K}=0.65$ [21] se obtiene un valor de $r=15.34$ $\mathrm{mm}$ y una relación de esbeltez $R_{\mathrm{ee}}=33.86$. A continuación, se calcula el valor de la relación de esbeltez de transición $C_{c}$ [17] con la ecuación (5), donde $S_{y}$ es el módulo de fluencia y $E$ es el módulo de elasticidad.

$$
C_{c}=\sqrt{\frac{2 E \pi^{2}}{S_{y}}}
$$

Para el acero $A 36$ se tiene $\mathrm{E}=200 \mathrm{GPa}$ [22] y $\mathrm{Sy}=\mathbf{2 5 0}$ MPa [21], obteniendo un valor $C_{C}=125.6$. En este caso la elación de esbeltez es menor a la relación de esbeltez de transición $\left(R_{e e}<C_{C}\right)$, clasificando el elemento BJ como columna corta [21]. A continuación se calcula la carga crítica $P_{\text {cr }}$ (o carga que la hará fallar por pandeo) mediante (6) [21], obteniendo $P_{c r}=90346.7 \mathrm{~N}$.

$$
P_{c r}=A S_{y}\left[1-\frac{S_{y}\left(K L / r_{\min }\right)^{2}}{4 \pi^{2} E}\right]
$$

Utilizando un factor de diseño, $n$, se puede calcular una carga admisible, $P_{a d m}$, a partir de (7) [17]. Con un factor de diseño $n=8$, la carga admisible sería
$P_{\text {adm }}=11293.3 \mathrm{~N}$, por lo cual la columna BL no fallaría por pandeo, dado que la carga actuante es de $582.9 \mathrm{~N}$. A partir de esto también se infiere que ningún elemento estructural presentará falla asociada a compresión o por el efecto de pandeo.

$$
P_{a d m}=\frac{P_{c r}}{n}
$$

A continuación se analizan los elementos de la parte inferior de la estructura, elementos $O P$ y $Q R$, encargados de soportar la carga ejercida por la bomba centrífuga. Para efectos de diseño se tomará la carga total generada por la bomba y se aplicará como una carga puntual en cada elemento como se indica en la figura 15 . Realizando un análisis estático, se han obtenido los valores para las reacciones en los apoyos, $R_{0}=74 \mathrm{~N}$ y $R_{p}=33.91 \mathrm{~N}$. Al ser el elemento QR similar al elemento OP, tanto en la distribución de la carga como en el valor de la carga, se tiene que $R_{0}=R_{Q}=74 \mathrm{~N}$ y $R_{P}=R_{R}=33.91 \mathrm{~N}$.

Los elementos LV y ST soportan la carga ejercida por el depósito de agua con un valor de 392.4N, para simplificar el cálculo se aproximará la anterior carga a $400 \mathrm{~N}$, y se omitirán las vigas WX y YZ. El depósito de agua tiene una longitud de profundidad de $0.4 \mathrm{~m}$, para una longitud de contacto de $0.8 \mathrm{~m}$ en ambas vigas. La carga generada por el depósito de agua se tomará como distribuida, para una carga total de $400 \mathrm{~N}$, resultando una carga distribuida de $500 \mathrm{~N} / \mathrm{m}$. La figura 16 muestra el diagrama de cuerpo libre del elemento LV. Aplicando un análisis estático, se han obtenido los valores para las reacciones en los apoyos, $R_{L}=100 \mathrm{~N}$ y $R_{V}=100 \mathrm{~N}$, al ser el elemento $\mathrm{ST}$ similar al elemento LV, tanto en la distribución de la carga como en el valor de la carga, se tiene que $R_{L}=R_{S}=R_{T}=R_{V}=100 \mathrm{~N}$.

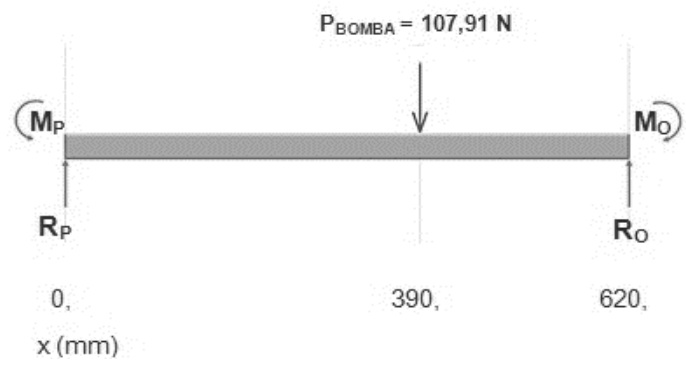

Figura 15. Diagrama de cuerpo libre elemento OP. 


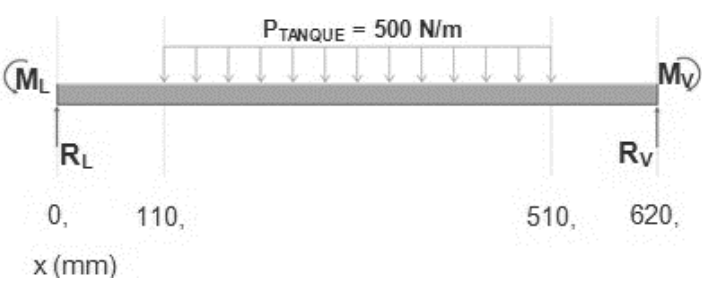

Figura 16. Diagrama de cuerpo libre elemento LV.

Dado que la viga del elemento IM soportará la mayor carga, en esta se encontrará el punto más crítico para toda la estructura. Esta viga es un caso especial por ser una viga doblemente empotrada. Se utilizará el método de doble integración para calcular los momentos en los apoyos. La figura 17(a) muestra el diagrama de cuerpo libre para el elemento IM. Las fuerzas que actúan directamente en los apoyos $\left(R_{A}\right.$ y $\left.R_{G}\right)$ se han omitido, debido a que no producen efecto directo sobre la viga. Para la suma total de momentos en $M_{1}=0$ (punto I) y aplicando el método de doble integración en vigas empotradas, se obtiene que $R_{1}=399.52 \mathrm{~N}$, $R_{M}=398.09 \mathrm{~N}, M_{1}=113.84 \mathrm{Nm}$ y $M_{M}=118.15 \mathrm{Nm}$. En la figura 17 (b) se observa el diagrama de cortantes y en la figura 17(c) el diagrama de momento flector para el elemento IM, construidos a partir de los valores de las cargas y los obtenidos en el análisis estático de dicho elemento. El momento flector máximo para la viga IM es de $118.15 \mathrm{Nm}$ en el apoyo M. A partir de este valor se calcula el mínimo factor de seguridad de la estructura, para determinar si la estructura soportará las cargas aplicadas. Para las vigas se utilizará el mismo perfil en tubo cuadrado de acero A36 de $40 \mathrm{~mm}$ con espesor de $2.5 \mathrm{~mm}$.

El esfuerzo, $\sigma$, se determina mediante (8) [21], para el tubo cuadrado se tiene un módulo de sección $\mathrm{S}=4.1 \mathrm{~mm}^{3}$ [23], y el momento máximo, $\mathrm{M}_{\max }$, fue determinado $M_{\max }=118.15 \mathrm{Nm}$ (figura 17), obteniendo un esfuerzo $\sigma=28.82 \mathrm{MPa}$. A partir de la ecuación (9) se calcula el factor de seguridad, $N$, [21], donde $S_{u}$ es límite elástico $\left(S_{u}=250 \mathrm{MPa}\right.$ para acero A36), y $\sigma$ fue calculado con la ecuación (8); se obtiene un factor de seguridad $\mathrm{N}=8.8$, concluyendo que este elemento (ni la estructura) fallará bajo las cargas establecidas.

$$
\begin{gathered}
\sigma=\frac{M_{\max }}{S} \\
N_{s}=\frac{S_{u}}{\sigma_{d}}
\end{gathered}
$$

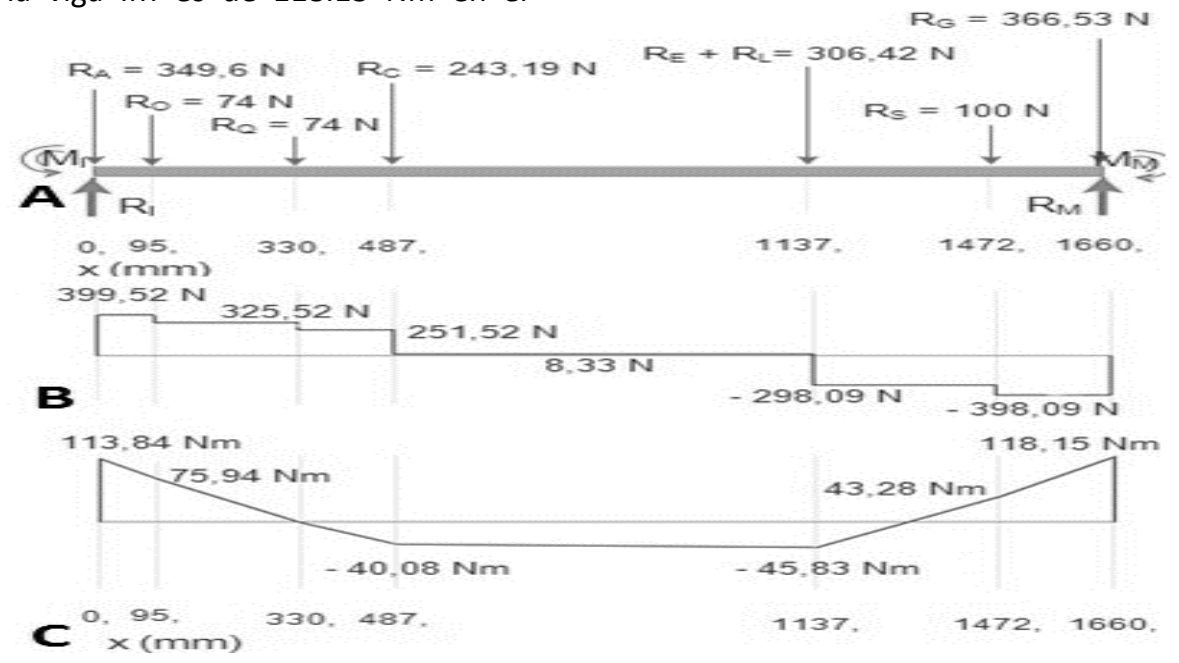

Figura 17. Diagramas elemento IM: (A) Cuerpo libre. (B) Fuerza cortante. (C) Momento flector.

\section{ANÁLISIS Y DISCUSIÓN DE RESULTADOS}

La verificación de los resultados se realiza con un análisis por elementos finitos usando SolidWorks, considerando las cargas y condiciones antes mencionadas. La figura 18(a) muestra el resultado obtenido en el análisis del esfuerzo, y la figura 18(b) muestra lo propio para el factor de seguridad. La simulación arroja un esfuerzo máximo de $25.87 \mathrm{MPa}$, un esfuerzo inclusive 
menor al obtenido con la ecuación (8). Similarmente para el factor de seguridad se obtiene 9.66 en la simulación, valor mayor al calculado mediante la ecuación (9), lo cual es favorable. Para el análisis de elementos finitos, el software incluye la mayor cantidad de variables y características reales del material como la geometría exacta de la estructura; caso contrario al cálculo estático donde se busca evaluar el diseño de una forma simplificada. Sin embargo, ambos resultados se asemejan, considerando entonces que el diseño es adecuado para el banco de pruebas y este no fallará así se apliquen cargas externas no convencionales. En la figura 19 se muestra el aspecto final del banco de pruebas.

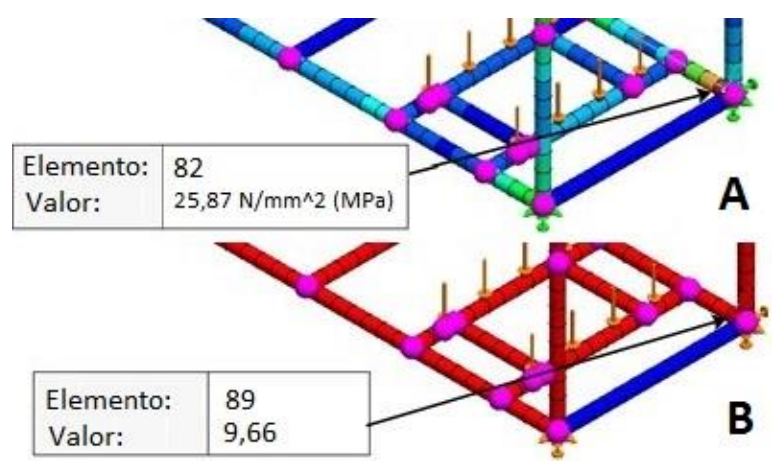

Figura 18. Análisis de elementos finitos. (A) Esfuerzo de diseño. (B) Factor de seguridad.

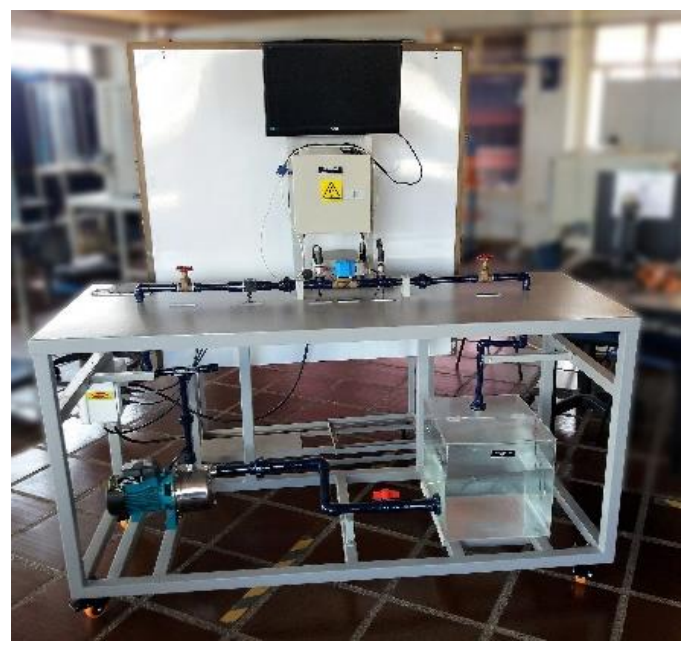

Figura 19. Aspecto final del banco de pruebas.

\section{CONCLUSIONES}

Mediante la combinación de diseño de producto bajo ergonomía fue posible el dimensionamiento de un equipo de entrenamiento, considerando también normativa de disposición de elementos de flujo. Tales criterios de ergonomía permitieron establecer dimensiones, alturas y áreas de trabajo, de acuerdo con un percentil de la población colombiana, tratando de proponer un producto que brinde comodidad a los usuarios finales. En cuanto al diseño estructural, se obtuvieron resultados satisfactorios al comparar resultados de simulación de elemento finito en SolidWorks, con los resultantes de teoría de falla. Se comparte de esta manera, en este artículo, una integración de conceptos para diseño de bancos de entrenamiento en ingeniería y profesiones afines.

\section{REFERENCIAS}

[1] P. Grech, Introducción a la Ingeniería. Un Enfoque a Través del Diseño. 2nd ed. Pearson Education, Colombia, 2013.

[2] G. Urrea, J. Niño, J. García, J. Alvarado, G. Barragán y O. Hazbón, "Del aula a la realidad. La importancia de los laboratorios en la formación del ingeniero" en WEEF World Engineering Education Forum Conf. Innovación en investigación y educación en ingeniería: factores clave para la competitividad local. pp. 2 [en línea]. Disponible en:

https://www.acofipapers.org/index.php/acofipape rs/2013/paper/viewFile/502/145 [Accedido: 27ene-2016]

[3] G. Lugo, "La importancia de los laboratorios", Construcción y Tecnología, vol. 223, pp. 20-21, dic. 2006.

[4] Asociación Colombiana de Ingenieros Eléctricos, Mecánicos y Afines ACIEM, "Áreas de formación de los ingenieros", ACIEM, vol. 1, no. 106, sep. 2006.

[5] A. Creus. Instrumentación Industrial, 8va. ed. México: Alfaomega grupo editor S.A, 2011.

[6] Industrial-Process Control Valves, IS/IEC 605342-3:1997.

[7] Verein Deutscher Ingenieure, "Fluidic Characteristics Quantities of Control Valves and Their Determination", Verband Der Elektrotechnik Elektronik Informationstechnik, Deutschland, VDI/VDE 2173, 2007.

[8] V. L. Karade, A. B. Shinde and P. V. Pol, "Control valve coefficient testing", International Journal of 
Innovative Research In Technology, IJIRT, vol. 2, pp. 199-203. nov. 2015.

[9] G. Aragón-Camarasa, G. Aragón-González, A. Canales and A. León, "Test bench for process control valves", in Fourth congress of electronics robotics and automotive mechanics, 2007, pp. 139144. DOI 10.1109/CERMA.2007.31

[10] GUNT Hamburg, RT 390 Test Stand for Control Valves, 2016. [Online]. Available: http://www.gunt.de/en/products/test-stand-forcontrol-valves/080.39000/rt390/glct-1:pa-148:pr1115?sccsf=1. [Accessed: Jan 10, 2016]

[11] "Válvulas solenoides servoaccionadas de 2/2 vías, tipo EV260B", DANFOSS, Nordborg, Dinamarca, 2013 [en línea]. Disponible en: http://files.danfoss.com/Technicallnfo/Dila/04/IC.P D.200.01.05.pdf [Accedido: 23-mar-2016]

[12] R. Mott. Mecánica de Fluidos. 6ta. ed. México: Pearson Educación, 2006.

[13] A. Lucszczewski, Redes industriales de tubería, bombas para agua, ventiladores y compresores: diseño y construcción, México: Reverté ediciones S.A de C.V., 1999.

[14] CRANE Co, Flujo de fluidos en válvulas, accesorios y tuberías. México: McGraw-Hill, 1992.

[15] E. Valdez. Abastecimiento de Agua Potable. 4ta. ed. México: Universidad nacional autónoma de México, 1994.

[16] "Bombas eléctricas", EDARVICO Eduardo Arango V. \& Cía. S. A., Cali, Colombia, 2016 [en línea]. Disponible en:

http://puntodeventa.co/\#/Bomba-Electrica

3/productos [Accedido: 05-abr-2016]

[17] International Ergonomics Association IEA, "Definition and Domains of ergonomics", 2016. [Online]. Available: http://www.iea.cc/whats/index.html. [Accessed: 02-feb-2016]

[18] J. Estrada, J. Camacho, M. Restrepo y C. Parra, "Parámetros antropométricos de la población laboral colombiana", Revista Facultad Nacional De Salud Pública, vol. 15, no. 2, p. 112-139. 1998.

[19] Universidad De Valencia. Prevención: “Diseño de Puestos de Trabajo. Curso online de Seguridad y Salud en el Trabajo,". Módulo 2, apartado 2.6.4, 2016, [en línea]. Disponible en: http://www.uv.es/sfpenlinia/cas/index.html.

[Accedido: 03-feb-2016]

[20] A. Rodríguez. Sistemas SCADA. 2da. ed. Barcelona: Marcombo S.A., 2007.
[21] R. Mott, Diseño de Elementos de Máquinas. 4ta. ed. México: Pearson Educación, 2006.

[22] F. P. Beer, E. R. Jhonston y J. T. Dewolf. Mecánica de Materiales, 3ra. ed, México: McGraw Hill interamericana S.A. de C.V, 2004.

[23] Tubos Colmena, "Perfil Estructural Tubular". 2016. [en línea]. Disponible en: http://www.tuboscolmena.com/web/fichas/Estruc tural_Cerrado.pdf. [Accedido: 25-abr-2016] 